odds of subsequent depressive symptoms (OR=2.17 (95\% CI) 1.65-2.84). Mediation analyses revealed that physical inactivity mediated the relationship between high CRP and depressive symptoms, explaining a total of $47.79 \%$ of this association.

Conclusion In this nationally representative sample, we found that physical inactivity is a partial mediator of the relationship between high CRP and subsequent depressive symptoms. Interventions targeting physical inactivity may be effective in ameliorating inflammation-associated depressive symptoms.

\section{OP62 A POPULATION BASED STUDY: ASSESSING MALE SUICIDE TRENDS AND INEQUALITIES IN SCOTLAND 1980-2015}

OR Molaodi*, D Brown, R Dundas, AH Leyland. MRC/CSO Social and Public Health Sciences Unit, University of Glasgow, Glasgow, UK

\subsection{6/jech-2018-SSMabstracts.61}

Background Male suicide rates increased remarkably in Scotland in the 1980s and 1990s, with higher rates in more deprived areas. We examine trends in male suicide in Scotland from 1980 to 2015 by method of suicide and individual socioeconomic position. We also explore trends in inequalities by individual socioeconomic position and the extent to which this may be attributable to suicide method.

Methods Suicide deaths for 1980-2015 were obtained from National Records Scotland. Inequalities were assessed using National Statistics socio-economic classification (NS-SEC) for ages 20-59 in 2000-2002 and 2010-2012. Standardised death rates were calculated by age with European population 2013 as reference, and Poisson regression was used to assess trend significance. Inequalities were assessed using Slope of Index of Inequality (SII).

Results There were 12281 suicide deaths between 1980 and 2015. No significant linear trend was observed over time for all ages, but suicide rates per 100000 person-years increased from 21 to 27 between 1980 and $2002 \quad(\mathrm{p}<0.001)$, and decreased from 27 to 20 from 2002 to 2015 (p<0.001). No significant trend was observed for poisoning, but suicide rate by hanging, suffocation and strangulation increased over time $(p<0.001)$, and decreased for other suicide $(p<0.001)$. There were significant differences in suicide rates between the NSSEC groups, and by suicide method (p-values $<0.001)$. SII: 85 (95\% CI, 77 to 92 ) in 2000-01 and 62 (95\% CI, 55 to 68) in 2010-12 per 100000 person-years, indicated that inequalities between social class extremes were significantly higher in 2000-02 than 2010-12. Suicide by hanging, suffocation and strangulation accounted for 44\% of inequalitiesin 2000-02 $(\mathrm{SII}=37 \quad(95 \% \mathrm{CI}, 33$ to 42$))$ and $49 \%$ in $2010-2012$ $(\mathrm{SII}=30(95 \% \mathrm{CI}, 25$ to 34$))$.

Conclusion The decline in male suicide rates may be attributed to suicide prevention strategies introduced by the Scottish Government from 2002 such as Choose Life. Despite decreasing trends of male suicide, suicide by hanging, suffocation, and strangulation increased over time. Inequalities by individual deprivation decreased between 2000-02 and 2010-12. Limitations are that NS-SEC categories, never worked and long term unemployed and not classified, were excluded from the analysis assessing inequalities since the interpretation of results for this group was ambiguous. What effect this omission would have on the estimation of inequalities is unclear. Caution should be taken when comparing inequalities by NSSEC 2001 and 2011 as in 2011 NS-SEC category was estimated for those without occupation. Policy should be directed at reducing deaths from hanging, suffocation, and strangulation.

\section{OP63 LONELINESS, LIVING ARRANGEMENTS AND EMOTIONAL SUPPORT AS PREDICTORS OF SUICIDALITY: A 7 YEAR FOLLOW-UP OF THE UK BIOBANK COHORT}

RJ Shaw*, B Cullen, N Graham, D Mackay, J Ward, R Pearsall, DJ Smith. Institute of Health and Wellbeing, University of Glasgow, Glasgow, UK

\subsection{6/jech-2018-SSMabstracts.62}

Background Between 1997 and 2017 the number of middleaged people living alone in the UK increased by $53 \%$ and loneliness is now recognised as an important policy area. We aimed to understand the interrelationships between loneliness, living arrangements and emotional support in predicting suicidal thoughts and behaviours.

Methods Between 2006 and 2010 sociodemographic and health data were collected from 500000 participants, aged 40-69, in UK Biobank. These data were linked to hospital admission records for self-harm and suicidal ideation until March 2015, and records for death by suicide until February 2016. Additionally, in 2016-2017, 150000 participants completed an online questionnaire which probed thoughts of selfharm, self-harm behaviour and attempted suicide. Exposures assessed were baseline measures of self-reported loneliness, living arrangements and emotional support (frequency of confiding). Deaths by suicide and hospital admissions were investigated with Cox proportional hazards models and logistic regression was used for self-report outcomes. Analyses were adjusted for socio-demographic factors including deprivation and employment, and multimorbidity.

Results In adjusted analyses loneliness was the risk most consistently related to all outcomes including death by suicide, (hazard ratio (HR) $1.75,95 \%$ CI 1.22 to 2.51), hospital admissions (HR 4.41, 95\% CI 2.50 to 7.76 ) and self-reported suicide attempts (HR 5.38, 95\% CI 3.35 to 8.63). After adjustment, not living with a partner was associated with increased risks of hospital admissions and dying by suicide, but not with the self-report measures of suicidality. Not living with a partner had a stronger relationship with death by suicide for men (HR 2.08, 95\% CI 1.36 to 3.18) than for women (HR 1.16, 95\% CI 0.59 to 2.31). After adjustment, emotional support was associated with the self-report but not administrative measures of suicidality. For example, when people with the least emotional support were compared to those with the most, the odds ratio was 3.00 (95\% CI 1.71 to 5.28). Statistical interactions indicated that people who were lonely despite living with a partner had the highest risk of contemplating self-harm and that loneliness eliminated the protective effects of living with a partner for deaths by suicide.

Conclusion Loneliness was the strongest predictor of suicide risk irrespective of living arrangements and emotional support and loneliness explained the relationships between living 